\title{
Intoxications aiguës par les bêta-bloquants : aspects cliniques et thérapeutiques
}

\section{Acute beta-blocker poisonings : clinical features and therapies}

\section{Bruno MEGARBANE*, Souheil KARYO, Frédéric BAUD}

Réanimation Médicale et Toxicologique, Hôpital Lariboisière, Université Paris VII, 2, rue Ambroise Paré - 75010 Paris

*Auteur à qui adresser la correspondance : Bruno MEGARBANE, Réanimation Médicale et Toxicologique, 2, rue Ambroise Paré - 75010 Paris - Tél : 0149958442 - Fax : 0149956578

E-mail : bruno-megarbane@wanadoo.fr

(Reçu le 20 septembre 2004 ; accepté après modifications le 26 octobre 2004)

\section{$R \hat{E} \boldsymbol{S U M E ́}$}

Le tableau clinique des intoxications par bêta-bloquants est dominé par les manifestations cardiovasculaires. Il s'agit le plus souvent d'une bradycardie sinusale et/ou d'une hypotension. Des troubles de conduction auriculo-ventriculaire voire intra-ventriculaire sont néanmoins possibles. L'hypotension artérielle résulte d'une baisse de la contractilité myocardique, à laquelle s'ajoute pour le labétalol, une vasodilatation, par effet alpha-bloquant. Au cours des formes sévères, apparaissent un coma, des convulsions, une dépression respiratoire et/ou des troubles métaboliques. La morbidité augmente significativement lorsque la molécule en cause possède des propriétés stabilisatrices de membrane ou lorsque d'autres cardiotropes sont co-ingérés. Le dosage plasmatique du bêta-bloquant est réalisable, mais sa valeur pronostique n'est pas établie. Le traitement doit être adapté à la gravité des troubles cardiovasculaires. La surveillance de la pression artérielle et de l'électrocardiogramme est suffisante chez les patients asymptomatiques. Le risque de complications est peu probable chez un patient resté asymptomatique, 6 heures après l'ingestion. L'absence d'accélération de la fréquence cardiaque sous atropine confirme le blocage adrénergique. En cas d'hypotension, le remplissage est prudent et la dobutamine l'antidote de choix. Le glucagon est efficace sur la pression artérielle, mais son effet chronotrope est plus modéré. L'adrénaline est l'option thérapeu-

\section{SUMMARY}

Cardiovascular signs usually dominate clinical features of beta-blocker acute poisonings. Most frequently, mild bradycardia or hypotension resumes the intoxication. However, exposures may sometimes result in the dysfunction of the atrioventricular or intraventricular conduction.

Hypotension is a consequence of cardiac contractility alteration and in case of labetolol poisoning, of an additive alpha-blockage-related vasodilatation. Coma, convulsions, respiratory depression and/or metabolic abnormalities may appear in the most severe poisonings. Morbidity significantly increases in case of membrane-stabilizing activity or coingestion of other cardioactive drugs. Plasma beta-blocker concentration measurement is now available, but its prognostic value is still unknown. Treatment should be adapted to the severity of cardiovascular signs. Clinical and ECG appear sufficient in asymptomatic patients. The risk of complication is low if the patient remains asymptomatic until 6 hours after the ingestion. The non-acceleration of heart rate after atropine infusion confirms the adrenergic blockage. In case of hypotension, fluid loading should be cautious and dobutamine represents the first-line antidote. Glucagon may be effective on blood pressure, but its chronotropic effect is moderate. Adrenaline is the therapeutic option for labetolol poisoning and isoprenaline for sotalol poisoning, due to the risk of torsades de pointe. Pacing may be necessary if high- 
tique pour les intoxications au labétalol et l'isoprénaline pour celles au sotalol, en raison du risque de torsade de pointe. La persistance de troubles de haut degré de la conduction auriculo-ventriculaire peut rendre nécessaire la mise en place d'un entraînement électro-systolique externe. L'apparition d'un collapsus d'origine cardiogénique, réfractaire aux catécholamines doit faire envisager le recours à l'assistance circulatoire externe.

\section{MOTS-CLÉS}

Intoxication aiguë, bêta-bloquant, effet stabilisant de membrane, cardiotrope, glucagon.

Les intoxications par bêta-bloquants sont plutôt rares, représentant 1-3\% des intoxications médicamenteuses aiguës admises dans un service de réanimation toxicologique (1). Aux Etats-Unis, 52156 cas d'exposition aux bêta-bloquants ont été rapportés sur une période d'observation de 11 années, dont $47 \%$ concernait l'ingestion de propranolol (2). Au cours des dernières années, l'incidence aurait tendance à augmenter, en raison de l'élargissement des indications de prescriptions de cette classe médicamenteuse : migraine, algie de la face, anxiété, ... La mortalité hospitalière reste cependant réduite en comparaison aux autres intoxications par cardiotropes, en raison notamment de l'efficacité de l'arsenal thérapeutique à disposition. Néanmoins, le risque de survenue d'intoxications sévères justifie de bien connaitre le tableau clinique, les facteurs pronostiques et les thérapeutiques symptomatiques et spécifiques.

\section{Propriétés toxicologiques des bêta-bloquants}

Les bêta-bloquants sont des antagonistes compétitifs des catécholamines endogènes ou de synthèse, au niveau des récepteurs bêta-adrénergiques (3). Ils sont classés en fonction de leur cardio-sélectivité, de leur activité sympathomimétique intrinsèque et de leur propriété alpha-bloquante (Tableau I) (4). Ils appartiennent à la classe II des anti-arythmiques de Vaughan- degree dysfunction of the atrioventricular conduction persists. If cardiac collapse is refractory to conventional therapies, extracorporeal life support may be indicated.

\section{KEY-WORDS}

Acute poisoning, beta-blocker, membrane stabilizing agent, cardiotropic drug, glucagon.

Williams et exercent sur le myocarde une action chronotrope, inotrope, dromotrope, et bathmotrope négative. Ils inhibent l'entrée du $\mathrm{Na}^{+}$et du $\mathrm{Ca}^{2+}$ à la phase 0 de la dépolarisation myocardique en réduisant la quantité d' $\mathrm{AMP}_{\mathrm{c}}$ intracellulaire. Leur action sur la contractilité est secondaire à l'inhibition de la libération du $\mathrm{Ca}^{2+}$ à partir du réticulum sarcoplasmique. Seul le sotalol possède des propriétés de la classe III des antiarythmiques, avec augmentation de la durée du potentiel d'action et de la période réfractaire, responsable d'un allongement de l'espace QT sur l'ECG. Certains bêta-bloquants sont à l'origine d'un effet stabilisant de membrane, avec un blocage des canaux sodiques responsable du courant sodique entrant dans les cellules au cours de la phase 0 du potentiel d'action. Il s'agit du propranolol, de l'acébutolol, du nadolol, du pindolol, du penbutolol, du labétalol, du métoprolol et de l'oxprénolol. Toutes ces molécules peuvent induire des manifestations cardiaques graves, mettant en jeu le pronostic vital, en raison du blocage de la conduction et de l'inhibition de l'inotropisme cardiaque.

À la suite de l'ingestion de doses élevées, des manifestations extra-cardiaques peuvent apparaître, liées à la perte de sélectivité cardiaque et à l'effet stabilisant de membrane (5). Ces manifestations peuvent également se rencontrer en cas d'interactions médicamenteuses ou de prédispositions individuelles, comme une maladie asthmatique. Les beta-bloquants peuvent en effet provoquer un bronchospasme, en levant le tonus bronchodilatateur à médiation bêta-adrénergique, notamment

Tableau I : Classification des bêta-bloquants en fonction de leur activité pharmacologique.

\begin{tabular}{|cc|cc|c|}
\hline \multicolumn{2}{|c|}{} & \multicolumn{2}{c|}{ Bêta-bloquants } \\
\hline \multicolumn{2}{|c|}{ Non-sélectifs } & \multicolumn{2}{c|}{ Cardio-sélectifs } & Avec activité alpha-bloquante \\
Avec ASI* & Sans ASI & Sans ASI & Avec ASI & \\
\hline pindolol** & nadolol** & aténolol & acébutolol & labétolol** \\
alprénolol & propranolol** & esmolol & céliprolol & bucindolol \\
oxprénolol** $^{* * *}$ & timolol & métoprolol & xamotérol & \\
penbutolo** & sotalol & bisoprolol & & \\
\hline
\end{tabular}

* activité sympathique intrinsèque

** effet stabilisant de membrane 
chez un sujet prédisposé. Ils peuvent également interagir avec le système rénine - angiotensine, la tension intraoculaire ainsi que les métabolismes lipidique et glucidique.

Les propriétés pharmacocinétiques des bêta-bloquants sont hétérogènes (3). La résorption digestive est excellente, notamment pour les molécules liposolubles et la distribution tissulaire rapide avec fixation préférentielle sur le cœur, le foie et les poumons. Les molécules les plus liposolubles (propranolol, alprénolol et bisoprolol) traversent le mieux les membranes biologiques, et notamment la barrière hémato-encéphalique, favorisant l'apparition d'effets neurologiques centraux. Le métabolisme dépend de l'effet de premier passage hépatique et est soumis à une grande variabilité interindividuelle. Pour les molécules hydrosolubles (nadolol, céliprolol, aténolol et sotalol), un plus faible effet de premier passage hépatique est compensé par une absorption intestinale plus réduite, donnant une biodisponibilité similaire aux molécules liposololubles. Le métabolisme hépatique de certains bêta-bloquants (acébutolol ou propranolol, par exemple) donne naissance à des métabolites actifs. L'élimination est préférentiellement biliaire pour les molécules liposolubles, et rénale pour les molécules les hydrophiles.

La durée des manifestations cliniques est fonction de la demi-vie d'élimination du produit, le plus souvent de moins de $72 \mathrm{~h}$. Elle peut être prolongée en cas d'ingestion de comprimés à libération prolongée, d'altération des fonctions d'élimination hépatique ou rénale. Il peut être néanmoins intéressant de savoir, qu'en l'absence de survenue d'un événement cardio-circulatoire significatif dans les 6 heures après l'ingestion d'un bêta-bloquant à libération immédiate, l'intoxication est le plus probablement bénigne (6).

\section{Facteurs pronostiques}

Les bêta-bloquants les plus souvent incriminés lors d'une ingestion volontaire sont le propranolol, l'aténolol, le métoprolol, le nadolol et le labétolol (2). La gravité des intoxications est directement corrélée à la nature et à la dose du bêta-bloquant ingéré (2,5-7). L'existence d'antécédents cardiovasculaires (hypertension artérielle, cardiopathie ischémique ou valvulaire) chez le sujet intoxiqué peut compliquer l'évolution. Dans environ $20 \%$ des cas, l'intoxication est monomédicamenteuse. Dans les autres cas, les toxiques associés sont des psychotropes, dont il faut tenir compte dans l'évaluation de l'état de conscience ou d'autres cardiotropes, entraînant alors une modification radicale du pronostic, notamment si le bêta-bloquant est associé à un inhibiteur calcique ou une molécule à effet stabilisant de membrane $(1,4)$. Une étude prospective améri- caine de 280 cas d'exposition aux beta-bloquants retrouvait en analyse multivariée que les 2 seuls facteurs prédictifs de morbidité cardiovasculaire était la co-ingestion de cardiotropes et l'effet stabilisant de membrane du bêta-bloquant ingéré (6). L'intérêt du dosage sanguin des bêta-bloquants, désormais disponible en urgence par des méthodes d'HPLC reste encore discuté $(8,9)$. Un groupe d'experts avait d'ailleurs établi en 2003 que le dosage des bêta-bloquants était utile pour le diagnostic différentiel mais que l'analyse toxicocinétique avait peu d'intérêt pour la prise en charge des patients (10).

\section{Manifestations cliniques}

Les manifestations cardiovasculaires dominent le tableau, par leur fréquence et leur gravité (4-6). Les intoxications asymptomatiques représentent 30 à $40 \%$ des intoxications, notamment chez le sujet sain, avec des molécules possédant un effet agoniste partiel (7). Les manifestations les plus fréquentes $(50 \%$ des cas) sont l'hypotension, la bradycardie et le bloc de conduction auriculo-ventriculaire (11). La clinique peut se résumer parfois à une bradycardie isolée (Figure 1) ou à une chute modérée de la pression artérielle, sans aucune manifestation d'hypoperfusion tissulaire. Les formes sévères (20\% des cas) se rencontrent surtout avec le propranolol $(2,6)$. Le tableau peut comporter un collapsus et des anomalies à l'ECG, des trois étages de naissance et de conduction de la dépolarisation cardiaque (12). L'effet stabilisant de membrane, que possèdent le propranolol ou l'acébutolol à fortes doses, se traduit par un élargissement des complexes QRS et un trouble de la repolarisation ventriculaire (aplatissement des ondes $\mathrm{T}$ et allongement de l'espace QT corrigé en fonction de la fréquence) (Figure 1). Cet effet est largement corrélé à la létalité de cette intoxication $(6,13,14)$. Par contre, le risque d'œè̀me aigu du poumon cardiogénique est minime, en l'absence d'arrêt circulatoire (15). Les manifestations neurologiques (coma et convulsions), rapportées surtout au propranolol, sont attribuables à la liposolubilité et à l'effet stabilisant de membrane de cette molécule $(3,16)$. La dépression respiratoire peut évoluer vers l'apnée mortelle, même en l'absence de trouble de conscience (17). Par contre, la survenue d'un bronchospasme est rare et semble réservée aux sujets prédisposés. L'hyperkaliémie est également rare $(<1 \%)(18)$, alors que l'hypokaliémie est secondaire au transfert de potassium vers le compartiment intracellulaire, en cas d'effet stabilisant de membrane. Un risque d'hypoglycémie a surtout été souligné chez le nourrisson lors d'intoxications accidentelles $(7,14)$.

L'intoxication au sotalol est plus rare mais peut provo- 
A

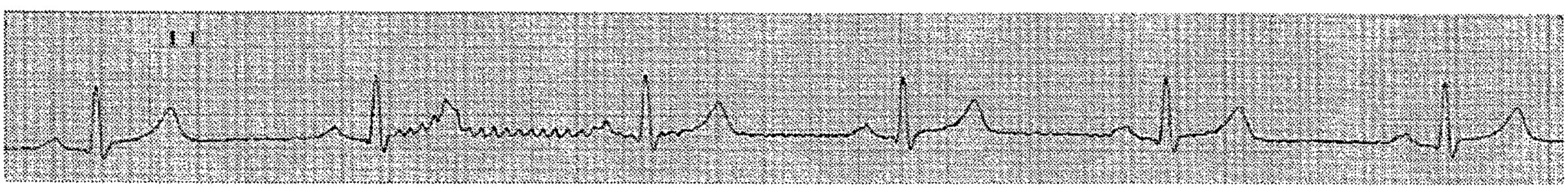

B

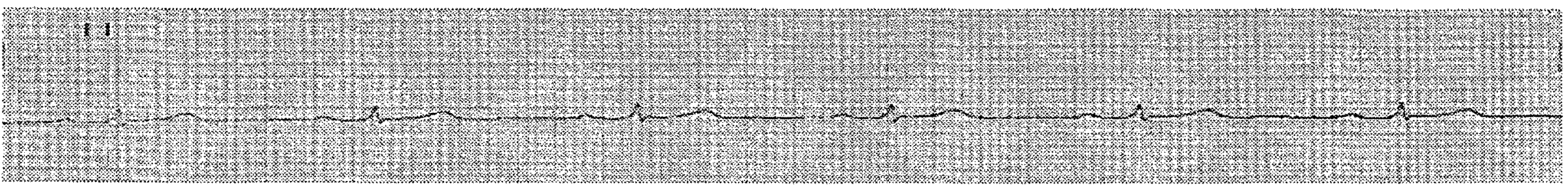

C

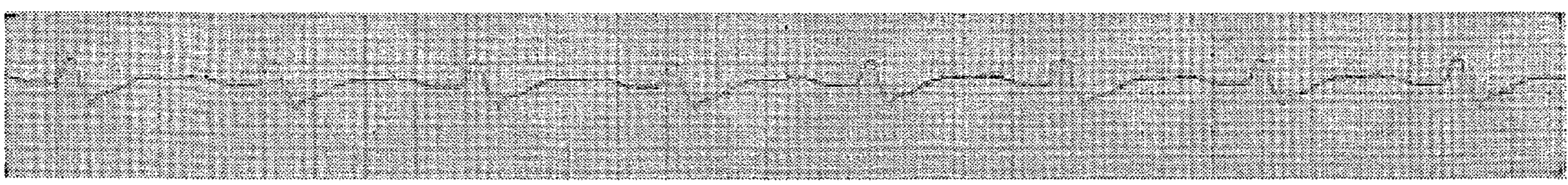

$\mathrm{D}$

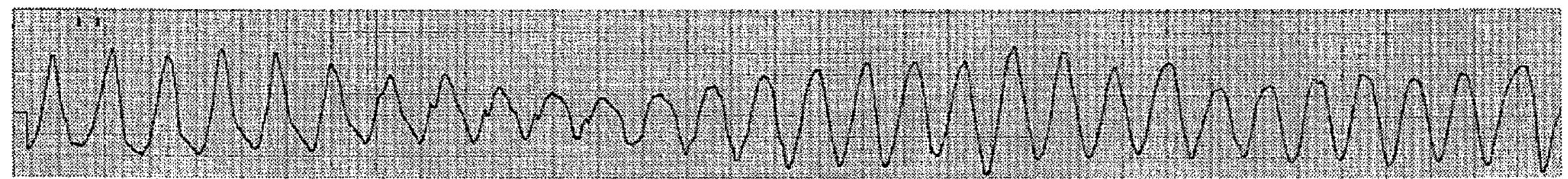

Figure 1 : Aspects ECG des intoxications par bêta-bloquants : bradycardie sinusale (A), bloc auriculo-ventriculaire (B), effet stabilisant de membrane avec bloc de conduction intra-ventriculaire $(C)$ et torsade de pointe $(D)$.

quer, en raison de ses propriétés pharmacologiques spécifiques une bradycardie à complexes QRS fins mais à intervalles QT allongés, exposant au risque de torsades de pointe et de tachycardie ventriculaire soutenue (19). Au cours de ces intoxications, la bradycardie n'est donc pas un signe de bénignité, puisqu'elle expose au risque de troubles du rythme mettant en jeu le pronostic vital.

\section{Prise en charge}

La décontamination digestive ne doit être faite que dans les 2 heures suivant l'ingestion, et en l'absence de contre-indications, dont notamment les troubles de la conscience (1). Elle ne doit jamais faire retarder les mesures symptomatiques ou antidotiques, seuls capables d'améliorer le pronostic du sujet intoxiqué. Le charbon activé est préféré au lavage gastrique, puisque les bêta-bloquants sont bien adsorbés par le charbon. Néanmoins, à ce jour, aucune étude n'a démontré un bénéfice clinique à la décontamination digestive, l'intérêt en cas d'ingestion de comprimés à libération prolongée restant encore à évaluer. Les méthodes d'épuration extra-corporelle (hémodialyse ou hémofiltration) sont peu utiles, en raison du large volume de distribution des bêta-bloquants et de leur liaison élevée aux protéines plasmatiques.

Au cours des intoxications sévères caractérisées par une dépression respiratoire, un collapsus ou des QRS élargies, la ventilation assistée doit être discutée rapidement, en parallèle au traitement pharmacologique (20). L'hypoxémie et l'acidose respiratoire potentialisent en effet la toxicité des bêta-bloquants et réduisent l'efficacité des catécholamines. Les autres indications de la ventilation doivent être discutées en cas de coma, de convulsions ou de bronchospasme sévère. Les troubles de la vigilance traduisent en général une baisse de la perfusion cérébrale, synonyme d'état de choc. L'intubation, après induction par une séquence rapide de l'anesthésie, permet d'éviter la majoration par l'hypoxie des troubles cardiaques et des conséquences neurologiques. La perfusion de bicarbonate molaire de sodium $(250 \mathrm{ml}$, à répéter si besoin, en fonction de l'affinement des QRS et à associer à $2 \mathrm{~g}$ de $\mathrm{KCl}$ ) est recommandée, en cas d'effet stabilisant de membrane, même si aucune étude, même expérimentale, n'a définitivement établie à ce jour son efficacité $(21,22)$.

Le traitement des troubles cardiovasculaires cherche à 
rétablir une fréquence cardiaque stable $\geq 60 /$ min et une pression artérielle systolique $\geq 100 \mathrm{~mm} \mathrm{Hg} \mathrm{(4).} \mathrm{Un} \mathrm{âge}$ avancé, une cardiopathie préexistante, en particulier hypertensive ou valvulaire aortique, incitent à une réévaluation à la hausse de ces paramètres. Devant une bradycardie, on commence par administrer $0,5 \mathrm{mg}$ d'atropine en IV directe. En cas de blocage adrénergique complet, l'atropine est incapable de provoquer l'accélération du cceur, après sa fixation compétitive aux récepteurs cholinergiques muscariniques. La correction d'une bradycardie par une dose unique d'atropine rend alors le diagnostic d'intoxication par betabloquants improbable (test diagnostique).

L'isoprénaline est utilisée de première intention en cas d'intoxication au sotalol, même en présence d'une bradycardie isolée sans hypotension, en raison du risque de torsade de pointes (19). Dans ces circonstances, la posologie nécessaire est habituellement comprise entre 1 et $5 \mathrm{mg} / \mathrm{h}$. Des doses plus importantes de l'ordre de 5 à $20 \mathrm{mg} / \mathrm{h}$ ont été nécessaires dans les premières heures. de traitement, notamment chez les sujets âgés souffrant de cardiopathie. Néanmoins, l'effet vasodilatateur $\beta_{2}+$ puissant de l'isoprénaline peut en limiter l'efficacité sur la pression artérielle et rendre nécessaire l'adjonction d'un agent vasoconstricteur. L'entraînement électrosystolique peut être proposé pour les blocs auriculoventriculaires de haut degré. Son efficacité est cependant modeste et les risques de complications mécaniques ou infectieuses en limitent les indications.

Le traitement de l'hypotension artérielle, définie par une pression systolique $<100 \mathrm{mmHg}$, débute par un remplissage vasculaire de $500 \mathrm{ml}$ de macromolécules ou de sérum salé isotonique. L'échec d'un traitement symptomatique justifie alors le recours aux antidotes, et les plus constamment efficaces sont la dobutamine, le glucagon, l'adrénaline et l'isoprénaline (Figure 2).

Le glucagon est un traitement classique des intoxications par bêta-bloquants, même si à ce jour, aucune étude clinique randomisée n'a établi son intérêt (23). Il est utilisé en cas d'hypotension, à la posologie initiale de $5-10 \mathrm{mg}(0,15 \mathrm{mg} / \mathrm{kg})$ en bolus IV, suivi, en cas d'efficacité, par une perfusion continue à la dose de 1-5 $\mathrm{mg} / \mathrm{h}(0,05-0,10 \mathrm{mg} / \mathrm{kg} / \mathrm{h})$, en raison de sa demi-vie courte de $20 \mathrm{~min}$. Il agit en court-circuitant la liaison du bêta-bloquant à son récepteur (Figure 3). Il permet d'obtenir une amélioration au moins partielle de la pression artérielle, grâce à son activité inotrope positive, alors que son action chronotrope est plus modeste. En dehors de nausées ou vomissements transitoires et d'une hyperglycémie modérée, le glucagon est bien toléré. La dilution des ampoules, dont la conservation se fait à $4^{\circ} \mathrm{C}$, se fait depuis 1998 dans un solvant fourni par le distributeur sans phénol, permettant d'éviter le risque théorique d'intoxication au phénol, jadis rappor- té à l'origine d'arythmies et d'hypotension (24). Les limitations importantes de cet antidote sont un coût élevé (environ 95 euros pour une perfusion de $5 \mathrm{mg} / \mathrm{h}$ pendant 24 heures) et un risque d'épuisement rapide du stock hospitalier, lors d'utilisation de fortes doses.

L'adrénaline est la catécholamine de choix en cas de collapsus persistant ou d'inefficacité de la dobutamine et du glucagon (25). Elle est préférée pour les intoxications par les alpha-bêta-bloquants, tel le labétalol. Utilisée en excès, elle peut exposer au risque d'élévation rapide des résistances systémiques, entrainant une baisse du débit cardiaque et un œdème pulmonaire cardiogénique, justifiant à ce stade, la nécessité d'une investigation hémodynamique par échocardiographie ou cathétérisme cardiaque droit.

La place de l'insuline euglycémique (insuline à forte doses $0,5 \mathrm{UI} / \mathrm{kg} / \mathrm{h}$ avec perfusion de glucose hypertonique), proposée au cours des intoxications graves par inhibiteurs calciques (26), n'est pas défini pour les intoxications par bêta-bloqueurs. Une étude expérimentale dans un modèle de chien intoxiqué par le propranolol a néanmoins suggéré la supériorité d'un tel protocole par rapport au glucagon ou à l'adrénaline (27).

Les inhibiteurs des phosphodiestérases (énoximone, amrinone) présentent des propriétés inotropes et vasodilatatrices intéressantes en cas d'insuffisance cardiaque à pression artérielle conservée (4). Ces molécules agissent en prévenant la dégradation de l'AMPc intra-cytoplasmique en 5'AMP. Leur utilisation n'est cependant pas de routine et n'est conseillée qu'en association au traitement conventionnel après appréciation du statut hémodynamique.

Des techniques d'assistances circulatoires externes, comme le ballon de contre-pulsion intra-aortique ou l'assistance par pompe centrifuge, ont été proposées, au cours d'intoxications massives avec état de choc cardiogénique, après échec des traitements médicaux conventionnels $(28,29)$. Au moins 7 cas d'assistance. circulatoire ont été rapportés dans la littérature (30). Plus récemment, la simplification du matériel d'assistance (pompe centrifuge et oxygénateur à membrane), la mise à disposition de canules percutanées adaptées et le perfectionnement de la technique d'abord chirurgicale des vaisseaux fémoraux permettent la prise en charge plus aisée des cas graves par des équipes médico-chirugicales entraînées. La meilleure connaissance des facteurs prédictifs de décès au cours des intoxications par bêta-bloquant avec effet stabilisant de membrane, permettrait d'envisager plus précocement ces thérapeutiques d'exception, pour éviter l'évolution vers l'encéphalopathie anoxique, séquellaire de l'hypoperfusion tissulaire. 


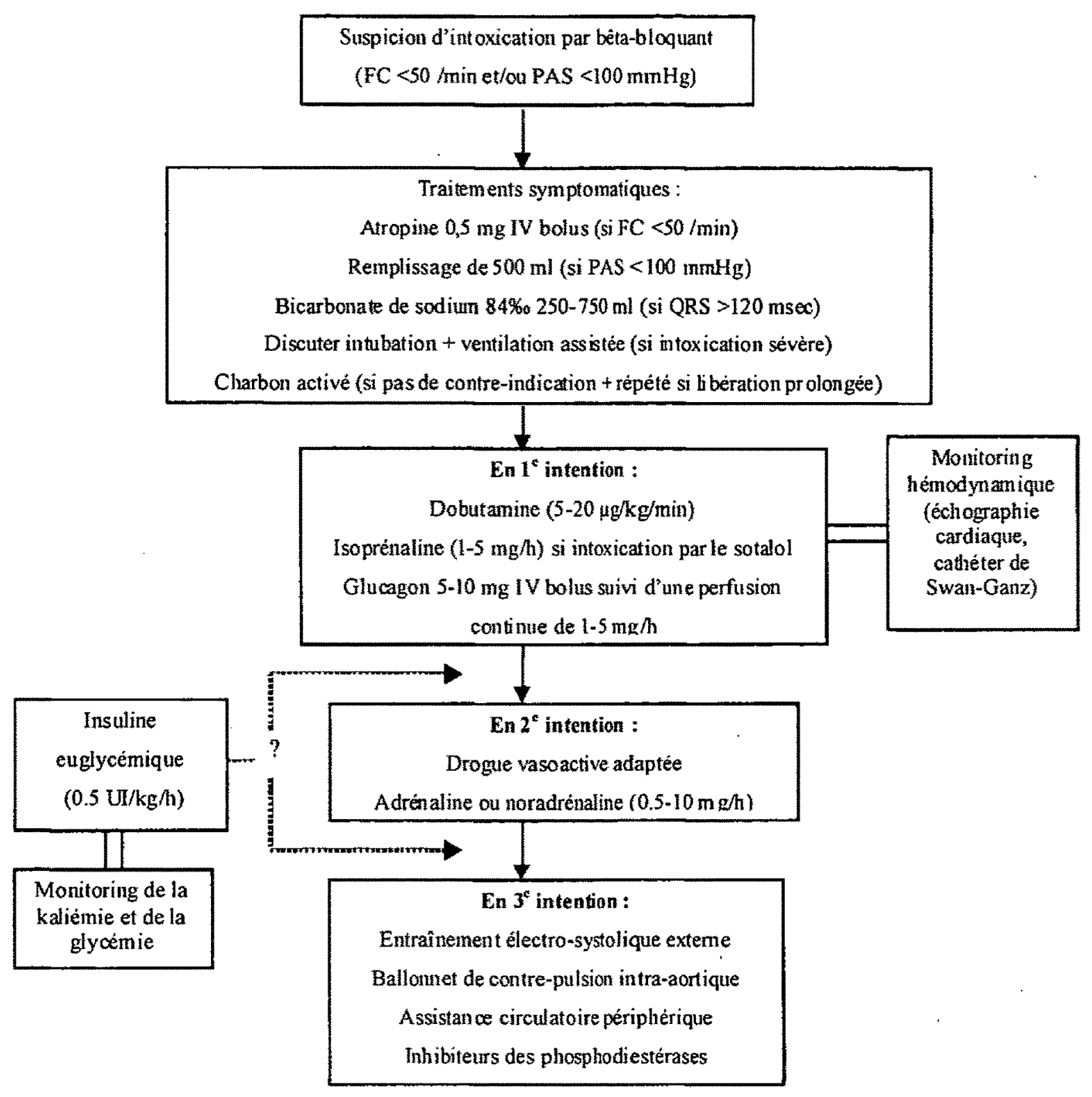

Figure 2 : Algorithme pour le traitement des intoxications par bêta-bloquants $(F C$ : fréquence cardiaque, PAS : pression artérielle systolique).

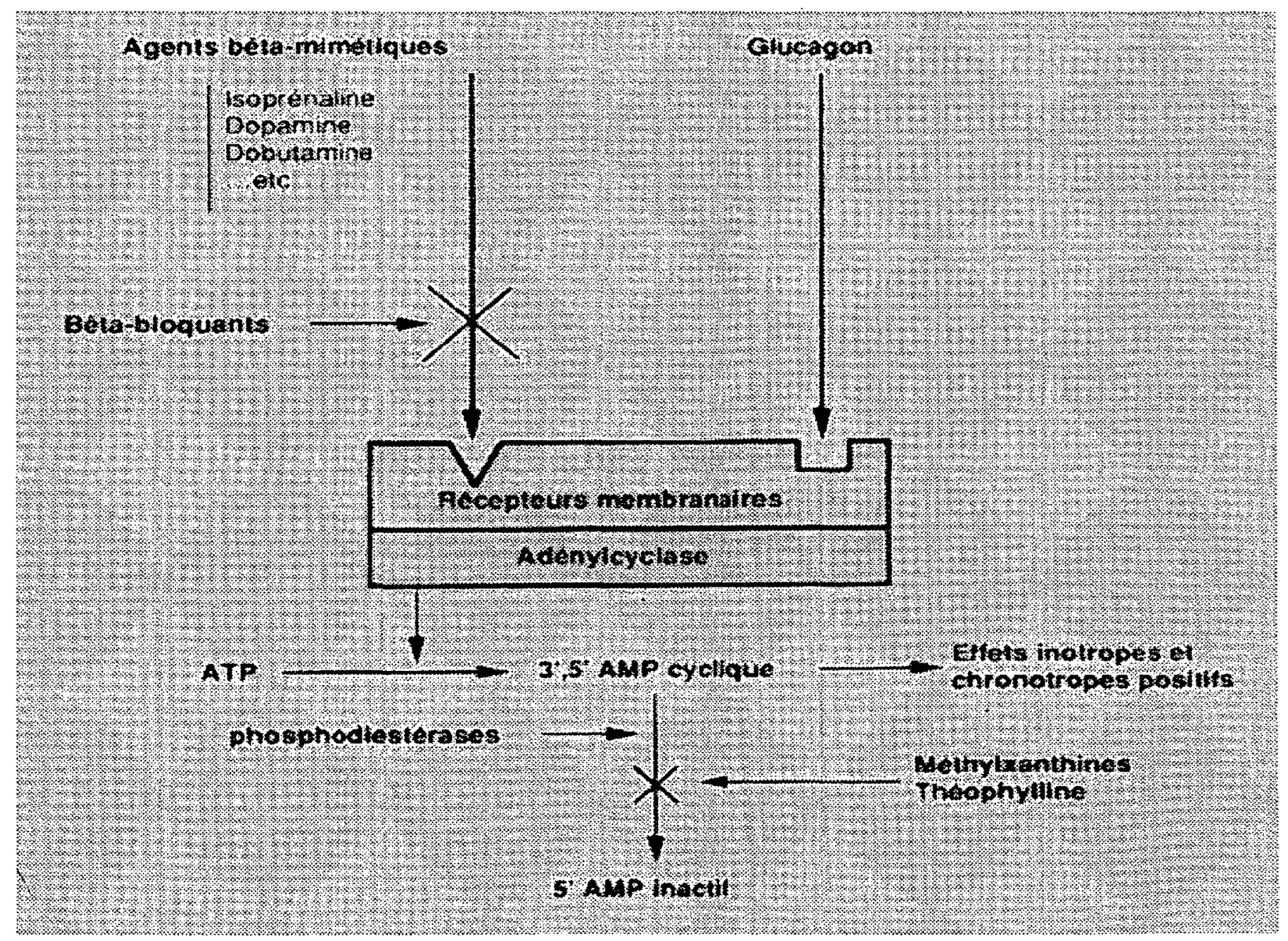

Figure 3 : Mécanisme d'action du glucagon au cours des intoxications par bêta-bloquant. 


\section{Conclusion}

Le tableau clinique d'une intoxication par bêta-bloquants est dominé par les manifestations cardio-circulatoires. Le pronostic est en général favorable, sauf en présence d'un toxique avec effet stabilisant de membrane ou de co-ingestion d'un autre cardiotrope, dont les inhibiteurs calciques. La faible mortalité hospitalière témoigne de la puissance de l'arsenal thérapeutique et de la possibilité de réversibilité des perturbations induites. La meilleure connaissance des facteurs pronostiques et notamment des facteurs prédictifs de décès pourrait permettre de recourir précocement, dans les cas les plus sévères, aux techniques d'assistance circulatoire périphérique.

\section{Références}

1. Mégarbane B., Baud F. Intoxications aiguës médicamenteuses. Encyclopédie Médico-chirurgicale, Toxicologie pathologie professionnelle, Editions Scientifiques et Médicales Elsevier SAS, 2002.

2. Love J.N., Litovitz T.L., Howell J.M., Clancy C. Characterization of fatal beta-blocker ingestion : a review of the American Association of Poison Control Centers data from 1985 to 1995. J. Toxicol. Clin. Toxicol. 1997 ; $35: 353-9$

3. Hoffman B.B., Lefkowitz R.J. Adrenergic receptor antagonist. In : Gilman A.G., Rall T.W., Niew A.S., Taylor P., eds. Goodman and Gilman's the pharmacological basis of therapeutics. 8th Ed. Pergamon Press, New York, 1990 ; 229-43.

4. Cariou A., Taboulet P. Réanimation des intoxications aiguës par bêta-bloquants. In : Baud $F$., ed. Réanimation des intoxications aiguës. Masson, Paris, 1995 ; 131-9.

5. Joye $\mathrm{F}$. Les intoxications aux bêta-bloquants. Presse Med. $2000 ; 29: 1027-33$.

6. Love J.N., Howell J.M., Litovitz T.L., Klein-Schwartz W. Acute beta blocker overdose : factors associated with the development of cardiovascular morbidity. J. Toxicol. Clin. Tox. $2000 ; 38: 275-81$.

7. Love JN, Sikka N. Are 1-2 tablets dangerous? Beta-blocker exposure in toddlers. J. Emerg. Med. 2004 ; $26: 309-14$.

8. Delothal Landes B., Megarbane B., Vieillard-Baron A. Recherche de cardiotropes en urgence ? Ann. Toxicol. Anal. $2002 ; 14: 148-9$ (abstract).

9. Delamoye M., Duverneuil C., Paraire F., de Mazancourt P., Alvarez J.C. Simultaneous determination of thirteen beta-blockers and one metabolite by gradient high-performance liquid chromatography with photodiode-array UV detection. Forensic Sci. Int. 2004 ; $141: 23-31$.

10. Goullet JP. Biomarqueurs de toxicité et anomalies métaboliques dans les principales intoxications graves. Symptomatologie clinique et toxique. Le prélèvement conservatoire. Ann. Bio. Clin. 2003; 61 : 421-33.

11. Love J.N. Beta-blocker toxicity : a clinical diagnosis. Am. J. Emerg. Med. $1994 ; 12: 356-7$.

12. Love J.N., Enlow B., Howell J.M., Klein-Schwarts W., Litovitz T.L. Electrocardiographic changes associated with beta-blocker toxicity. Ann. Emerg. Med. $2002 ; 40$ : 603-10.
13. Joye F., Harry P., Ferrandière M., Perrotin D. L'élargissement du QRS : un critère de gravité imminente dans les intoxications aux bêta-bloquants. Presse Med. $2000 ; 29: 142-3$.

14. Reith D.M., Dawson A.H., Epid D., Whyte I.M., Buckley N.A., Sayer G.P. Relative toxicity of beta blockers in overdose. J. Toxicol. Clin. Toxicol. 1996 ; 34 : 273-8.

15. Taboulet P., Cariou A., Berdeaux A., Bismuth C. Pathophysiology and management of beta-blocker self poisoning. J. Toxicol. Clin. Toxicol. 1993 ; 31 : 531-52.

16. Lifshitz M., Zucker N., Zalzstein E. Acute dilated cardiomyopathy and central nervous system toxicity following propranolol intoxication. Pediatr. Emerg. Care $1999 ; 15: 262-3$.

17.Langemeijer J.J.M, De Wildt D.J., De Groot G., Sangsster B. Centrally induced respiratory arrest: a main cause of death in B-adrenoceptor antagonist intoxication. Human Toxicol. $1986 ; 5: 65$.

18. Delacour J.L., Blanc P.L., Wagschal G., Daoudal P. L'hyperkaliémie dans l'intoxication aiguë aux bêta-bloquants. Presse Med. $1986 ; 15: 1377$.

19. Beattie J.M. Sotalol induced torsade de pointes. Scott. Med. J. $1984 ; 29: 240-4$.

20. Köppel C. Intoxications par les bêta-bloquants. In : Jaeger A, Vale JA, eds. Intoxications aiguës. Elsevier, Paris, $1999 ; 214-22$.

21. Love J.N., Howell J.M., Newsome J.T., Skibbie D.F., Dickerson L.W., Henderson K.J. The effect of sodium bicarbonate on propranolol-induced cardiovascular toxicity in a canine model. J. Toxicol. Clin. Toxicol. 2000 ; $38: 421-8$.

22. Donovan K.D., Gerace R.V, Dreyer J.F. Acebutololinduced ventricular tachycardia reversed with sodium bicarbonate. J. Toxicol. Clin. Toxicol. 1999 ; 37 : 481-4.

23. Bailey B. Glucagon in beta-blocker and calcium channel blocker overdoses: a systematic review. J. Toxicol. Clin. Toxicol. $2003 ; 41:$ 595-602.

24. Burda AM, Kapustka CA. Reformulated glucagon diluent phenol-free. J. Toxicol. Clin. Toxicol. $1999 ; 37: 127$.

25. Hicks P.R., Rankin A.P. Massive adrenaline doses in labetalol overdose. Anaesth. Intensive Care $1991 ; 19: 447-9$.

26. Yuan T.H., Kerns W.P. 2nd, Tomaszewski C.A., Ford M.D., Kline J.A. Insulin-glucose as adjunctive therapy for severe calcium channel antagonist poisoning. $\mathrm{J}$. Toxicol. Clin. Toxicol. $1999 ; 37: 463-74$.

27. Kerns W. 2nd, Schroeder D., Williams C., Tomaszewski C., Raymond R. Insulin improves survival in a canine model of acute beta-blocker toxicity. Ann. Emerg. Med. $1997 ; 29: 748-57$.

28. Lane A.S., Woodward A.C., Goldman M.R. Massive propranolol overdose poorly responsive to pharmacologie therapy : use of the intra-aortic balloon pump. Ann. Emerg. Med. $1987 ; 16: 1381-3$.

29. Babatasi G., Massetti M., Verrier V., Lehoux P., Le Page O., Bruno P.G., Khayat A. Intoxication sévère par médicaments cardiotoxiques : intérêt de l'assistance cardiocirculatoire percutanée en urgence. Arch. Mal. Cœur 2001 ; $94: 1386-92$.

30. Bosquet C., Jaeger A. Thérapeutiques d'exception au cours des défaillances circulatoires et respiratoires d'origine toxique. Réanimation $2001 ; 10: 402-11$ 